\title{
Hepatocellular carcinoma recurrence in HCV patients treated with direct-acting antivirals after curative treatment
}

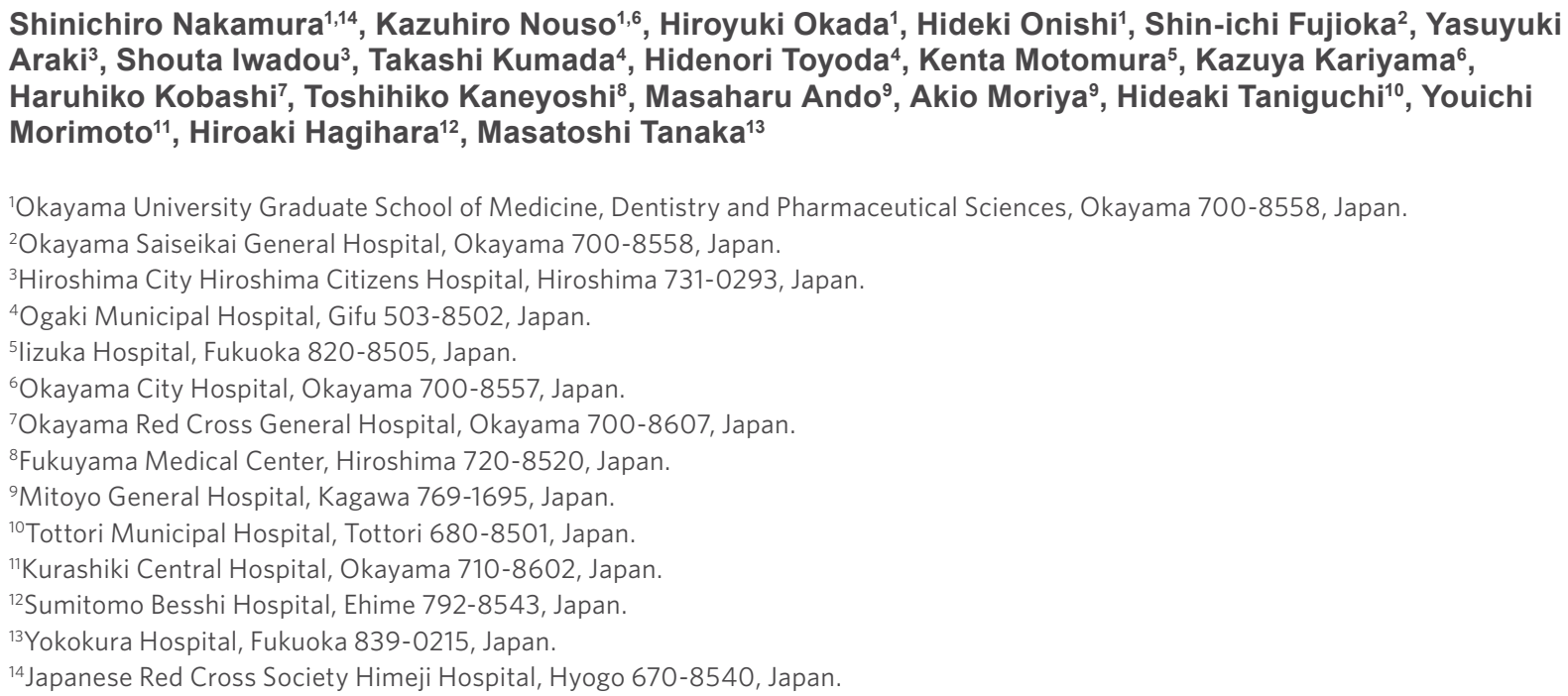

Shinichiro Nakamura'1,14, Kazuhiro Nouso ${ }^{1,6}$, Hiroyuki Okada1, Hideki Onishi', Shin-ichi Fujioka², Yasuyuki Araki $^{3}$, Shouta Iwadou ${ }^{3}$, Takashi Kumada ${ }^{4}$, Hidenori Toyoda ${ }^{4}$, Kenta Motomura ${ }^{5}$, Kazuya Kariyama ${ }^{6}$, Haruhiko Kobashi ${ }^{7}$, Toshihiko Kaneyoshi ${ }^{8}$, Masaharu Ando ${ }^{9}$, Akio Moriya ${ }^{9}$, Hideaki Taniguchi ${ }^{10}$, Youichi Morimoto $^{11}$, Hiroaki Hagihara ${ }^{12}$, Masatoshi Tanaka ${ }^{13}$

'Okayama University Graduate School of Medicine, Dentistry and Pharmaceutical Sciences, Okayama 700-8558, Japan.

${ }^{2}$ Okayama Saiseikai General Hospital, Okayama 700-8558, Japan.

${ }^{3}$ Hiroshima City Hiroshima Citizens Hospital, Hiroshima 731-0293, Japan.

${ }^{4}$ Ogaki Municipal Hospital, Gifu 503-8502, Japan.

${ }^{5}$ lizuka Hospital, Fukuoka 820-8505, Japan.

${ }^{6}$ Okayama City Hospital, Okayama 700-8557, Japan.

7Okayama Red Cross General Hospital, Okayama 700-8607, Japan.

${ }^{8}$ Fukuyama Medical Center, Hiroshima 720-8520, Japan.

${ }^{9}$ Mitoyo General Hospital, Kagawa 769-1695, Japan.

${ }^{10}$ Tottori Municipal Hospital, Tottori 680-8501, Japan.

${ }^{11}$ Kurashiki Central Hospital, Okayama 710-8602, Japan.

${ }^{12}$ Sumitomo Besshi Hospital, Ehime 792-8543, Japan.

${ }^{13}$ Yokokura Hospital, Fukuoka 839-0215, Japan.

${ }^{14}$ Japanese Red Cross Society Himeji Hospital, Hyogo 670-8540, Japan.

Correspondence to: Dr. Kazuhiro Nouso, Department of Gastroenterology and Hepatology, Okayama University Graduate School of Medicine, Dentistry and Pharmaceutical Sciences, 2-5-1 Shikata-cho, Kita-ku, Okayama 700-8558, Japan. E-mail: nouso@cc.okayama-u.ac.jp

\begin{abstract}
How to cite this article: Nakamura S, Nouso K, Okada H, Onishi H, Fujioka S, Araki Y, Iwadou S, Kumada T, Toyoda H, Motomura K, Kariyama K, Kobashi H, Kaneyoshi T, Ando M, Moriya A, Taniguchi H, Morimoto Y, Hagihara H, Tanaka M. Hepatocellular carcinoma recurrence in $\mathrm{HCV}$ patients treated with direct-acting antivirals after curative treatment. Hepatoma Res 2019;5:16.

http://dx.doi.org/10.20517/2394-5079.2019.06
\end{abstract}

Received: 21 Jan 2019 First Decision: 13 Mar 2019 Revised: 15 Mar 2019 Accepted: 17 Apr 2019 Published: 14 May 2019

Science Editor: Guang-Wen Cao Copy Editor: Cai-Hong Wang Production Editor: Huan-liang Wu

\begin{abstract}
Aim: The increased risk of hepatocellular carcinoma (HCC) recurrence in hepatitis $\mathrm{C}$ virus (HCV)-infected patients treated with direct-acting antivirals (DAAs) after curative treatment for HCC is controversial. The purpose of this study was to examine the risk of HCC recurrence after DAA therapy.
\end{abstract}

Methods: We conducted a retrospective cohort study of 312 consecutive patients with HCV-related HCC who received DAA therapy in participating institutions between September 2014 and July 2016. All patients received curative 
hepatectomy or radio-frequency ablation. We calculated the annual incidence of HCC recurrence after DAA therapy and identified the risk factors for HCC recurrence using Cox regression models.

Results: The median age was 74 years old, and a sustained virological response was achieved by 288 patients. The 3-year-overall survival rate was $95.4 \%$ in a median follow-up period of 855 days. HCC recurred in 135 patients. The $1-, 2$ - and 3-year recurrence rates were $18.3 \%, 38.8 \%$ and $55.4 \%$, respectively. A multivariate analysis revealed that the following factors were associated with HCC recurrence: multiple tumors at the first HCC treatment [hazard ratio (HR) $=2.21 ; 95 \% \mathrm{Cl}: 1.41-3.49]$, a history of multiple treatments for $\mathrm{HCC}(\mathrm{HR}=1.97 ; 95 \% \mathrm{Cl}: 1.28-3.02)$, and $\alpha$-fetoprotein (AFP-L3) $\geq 10 \%$ at the initiation of DAA therapy (HR $=4.74 ; 95 \% \mathrm{Cl}: 2.10-10.7)$.

Conclusion: Among patients treated with DAAs after the curative treatment of HCC, multiple tumors at the first HCC treatment, multiple prior HCC treatments and a high AFP-L3 level before DAA therapy were associated with recurrence, and the rate of recurrence was comparable to that before the DAA era.

Keywords: Hepatocellular carcinoma, hepatitis C virus, direct-acting antiviral, recurrence

\section{INTRODUCTION}

Worldwide, primary liver cancer is the second and sixth leading cause of cancer mortality in men and women, respectively ${ }^{[1,2]}$. The most frequent cause of HCC is liver cirrhosis due to HCV infection ${ }^{[3]}$.

DAA therapy have made it possible for most patients with HCV infection to achieve a sustained virological response, even if they cannot tolerate interferon-based therapy. The introduction of DAA therapy is expected to improve the prognosis of patients with liver cirrhosis due to HCV infection, and it is also expected that the recurrence rate will decrease in patients after HCC treatment. However, recent studies have suggested that DAA therapy might increase the risk of HCC recurrence ${ }^{[4,5]}$. For example, a Spanish multicenter study reported by Reig et al ${ }^{[4]}$ warned that DAA therapy may increase the risk of HCC recurrence. In their paper, 16/58 (27.6\%) patients who received DAA therapy after HCC treatment experienced tumor recurrence after a median follow-up period of 5.7 months. Subsequently, several studies reporting contradictory findings have been published ${ }^{[6-15]}$.

Although DAAs have been demonstrated to lower carcinogenicity in patients without a history of HCC treatment ${ }^{[8,16]}$, the effect of DAAs for preventing recurrence after HCC treatment has not been proven. In this study, we investigated the outcomes of HCC patients who received DAA therapy after curative treatment with hepatectomy or radio-frequency ablation (RFA) in a multicenter collaborative study and attempted to elucidate the effect of DAAs on recurrence.

\section{METHODS}

\section{Patients}

We performed a multicenter, retrospective cohort analysis of HCC patients who had previously been treated with hepatectomy or RFA and who received anti-HCV treatment with DAAs between September 2014 and July 2016. In this study, 312 consecutive patients were enrolled: 224 (71.8\%) and 88 (28.2\%) patients received RFA and hepatectomy before DAA treatment, respectively. A flowchart of the patient selection is shown in the Supplementary Figure 1.

All patients were diagnosed as cancer-free prior to DAA treatment based on triple-phase multidetector computed tomography (CT), dynamic contrast-enhanced magnetic resonance imaging (MRI) or ultrasonography (US). We confirmed the cancer-free status at least with two imaging modalities. The study protocol conformed to the ethical 


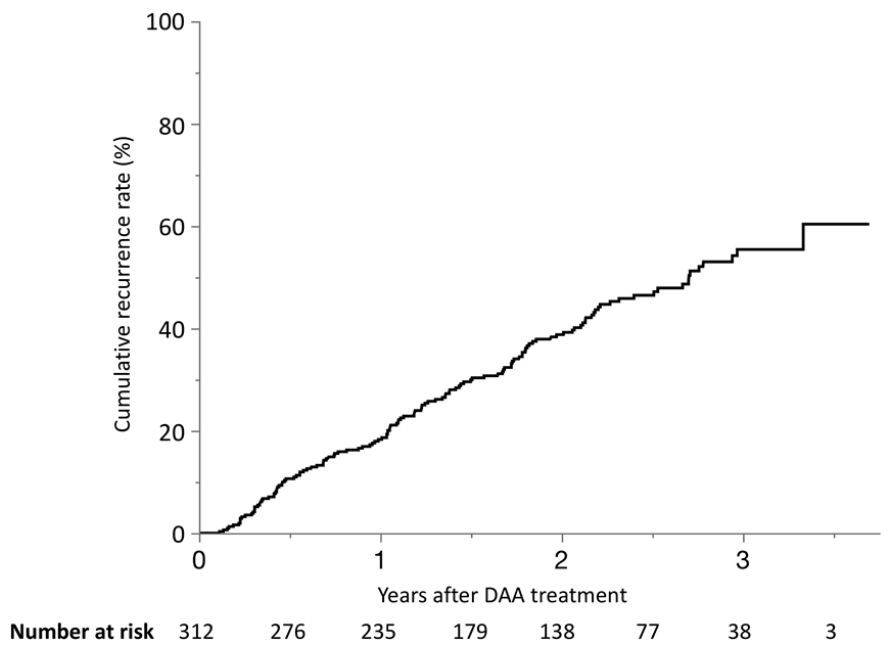

Figure 1. The cumulative recurrence rate in patients treated with DAAs after curative treatment for HCC. HCC recurred in 135 patients. The 1-, 2- and 3-year cumulative recurrence rates after DAA therapy were 18\%, 39\% and 55\%, respectively. HCC: hepatocellular carcinoma; DAA: direct-acting antiviral

guidelines of the World Medical Association and the Declaration of Helsinki and was approved by the ethics committees of each institute.

\section{DAA treatment}

The DAA regimens were daclatasvir/asnaprevir $(n=130,41.7 \%)$, sofosbuvir/ledipasvir $(n=116,37.2 \%)$, sofosbuvir/ ribavirin $(n=61,19.6 \%)$ and ombitasvir/paritaprevir/ritonavir $(n=5,1.6 \%)$. We assessed the response to DAA treatment based on the presence of HCV-RNA at 12 weeks after the end of the treatment. Patients negative for HCV-RNA at this time were considered to have achieved a sustained virological response (SVR12). The presence of HCV-RNA was examined using real-time polymerase chain reaction.

\section{Follow-up}

After DAA treatment, the patients were assessed every three months by US, triple-phase CT, or MRI. The diagnosis of HCC recurrence was confirmed via typical HCC imaging patterns obtained by angiography, CT, MRI and US. The criteria for the imaging-based diagnosis of HCC have been described in previous reports: hyper-attenuation at the hepatic arterial phase, hypo-attenuation at the portal venous phase in triple-phase CT or MRI and tumor staining on angiography, or hyper-enhancement in the arterial phase and hypo-enhancement in the portal venous and late phases on contrast-enhanced $\mathrm{US}^{[17,18]}$.

\section{Statistical analyses}

The clinical characteristics of the patients were obtained at the initial treatment for HCC and before and after DAA treatment. The data collected included the age, gender, platelet count, albumin (ALB), total bilirubin (T.Bil), aspartate aminotransferase (AST), alanine aminotransferase (ALT), gamma-glutamyl transpeptidase (GGTP), prothrombin time-international normalized ratio, Child-Pugh grade, AFP, des- $\gamma$-carboxy prothrombin (DCP), lens culinaris agglutinin-reactive AFP-L3, fibrosis (FIB-4) index, HCV genotype, amount of HCV-RNA, history of HCC treatment, number of tumors, maximum diameter of the tumor, date of HCC recurrence, treatment methods and survival.

Patients without recurrence were censored at the last visit or on the date of death. We calculated the annual HCC recurrence rate after DAA therapy and examined the risk factors for recurrence using Cox proportional hazard models. Variables with a $P$ value of $\leq 0.1$ in a univariate analysis were incorporated into a multivariate analysis by stepwise selection. The recurrence rate and overall survival rate after DAA therapy were calculated using the Kaplan-Meier method and evaluated with a log-rank test. $P$ values of $<0.05$ were considered to indicate statistical significance. All analyses were performed using the JMP Pro 14.1.0 software package (SAS Institute, Inc., Cary, NC, USA). 
Table 1. The baseline characteristics of 312 patients at the time of direct-acting antiviral treatment

\begin{tabular}{|c|c|c|c|}
\hline \multirow{2}{*}{ Characteristics } & \multirow{2}{*}{ All patients $(n=312)$} & \multicolumn{2}{|c|}{ Final HCC Tx before DAA } \\
\hline & & Hepatectomy $(n=89)$ & RFA $(n=223)$ \\
\hline Age (years) & $74(68-79)$ & $74(68-78)$ & $74(68-79)$ \\
\hline Gender (male) & $182(58.3 \%)$ & $50(56.2 \%)$ & $132(59.1 \%)$ \\
\hline Platelet $\left(\times 10^{3} / \mu \mathrm{L}\right)^{\star}$ & $105(76-140)$ & $113(98-137)$ & $99(73-142)$ \\
\hline $\operatorname{ALB}(g / d L)^{\star}$ & $3.8(3.4-4.0)$ & $3.9(3.6-4.2)$ & $3.7(3.4-4.0)$ \\
\hline T.Bil $(\mathrm{mg} / \mathrm{dL})^{\star \star}$ & $0.8(0.6-1.1)$ & $0.73(0.6-0.9)$ & $0.89(0.6-1.2)$ \\
\hline AST (U/L) & $52(37-67)$ & $51(39-70)$ & $52(36-66)$ \\
\hline $\operatorname{ALT}(U / L)$ & $41(28-60)$ & $42(31-65)$ & $39(27-60)$ \\
\hline $\operatorname{GGTP}(U / L)$ & $37(25-55)$ & $40(26-62)$ & $36(24-52)$ \\
\hline PT-INR & $1.07(1.0-1.14)$ & $1.07(0.99-1.11)$ & $1.06(1.00-1.15)$ \\
\hline Child-Pugh grade $A^{\star \star}$ & $280(89.7 \%)$ & $83(93.3 \%)$ & $197(88.3 \%)$ \\
\hline Fib-4 index & $5.64(3.75-8.04)$ & $5.06(3.65-6.85)$ & $5.88(3.75-8.53)$ \\
\hline \multicolumn{4}{|l|}{ HCV genotype $^{\star}$} \\
\hline Genotype 1 & $251(80.5 \%)$ & $65(73.0 \%)$ & $186(83.4 \%)$ \\
\hline Genotype 2 & $60(19.2 \%)$ & $24(27.0 \%)$ & $36(16.2 \%)$ \\
\hline Genotype $1+2$ & $1(0.3 \%)$ & $0(0 \%)$ & $1(0.4 \%)$ \\
\hline HCV-RNA (log IU/mL) & $6.0(5.4-6.4)$ & $6.0(5.4-6.5)$ & $6.0(5.4-6.4)$ \\
\hline SVR at 12 weeks & $288(92.3 \%)$ & $83(93.3 \%)$ & 205 (91.9\%) \\
\hline
\end{tabular}

${ }^{\star} P<0.001 ;{ }^{*} P<0.05$; The values indicate the median (interquartile range) unless otherwise noted. ALB: albumin; T.Bil: total bilirubin; AST: aspartate aminotransferase; ALT: alanine aminotransferase; GGTP: gamma-glutamyl transpeptidase; PT-INR: prothrombin time-international normalized ratio; FIB-4 index = Age (years) $\times$ AST $(\mathrm{U} / \mathrm{L}) /\left[\mathrm{PLT}\left(10^{9} / \mathrm{L}\right) \times \mathrm{ALT}^{1 / 2}(\mathrm{U} / \mathrm{L})\right]$; HCV: hepatitis C virus; HCC: hepatocellular carcinoma; Tx: treatment; SVR: sustained virological response

\section{RESULTS}

\section{Patient characteristics}

The clinical characteristics of the patients and the characteristics of HCC before DAA treatment are shown in [Tables 1 and 2], respectively. The median age was 74 years old, and $182(58.3 \%)$ patients were male. Two hundred and fifty-one (80.4\%) and 60 patients (19.2\%) had HCV genotypes 1 and 2, respectively, and 1 patient had both. An SVR12 was achieved by 288 patients $(92.3 \%)$. The median tumor size at the initial treatment was $18 \mathrm{~mm}$, and 244 patients $(78.2 \%)$ had a single tumor. The interval from the final HCC treatment and DAA therapy was 297 days (median). The median follow-up time from the end of DAA treatment was 855 days.

\section{The recurrence and survival rates}

HCC recurred in 135 patients (43.2\%), and the 1-, 2- and 3-year cumulative recurrence rates after DAA therapy were $18.3 \%, 38.8 \%$ and $55.4 \%$, respectively [Figure 1]. The 1-, 2 - and 3 -year-overall survival rates were $99.4 \%, 98.6 \%$ and $95.4 \%$, respectively [Figure 2 ].

\section{Risk factors for recurrence}

In the univariate analysis, the factors associated with HCC recurrence included AFP $\geq 10 \mathrm{ng} / \mathrm{mL}$ and multiple tumors at the initial HCC treatment, T.Bil $>0.8 \mathrm{mg} / \mathrm{dL}$, AFP-L $3 \geq 10 \%$ at the initiation of DAA therapy, a history of multiple treatments before DAA therapy, RFA as the final HCC treatments before DAA, period between the final HCC treatment and DAA therapy $<1$ year, AFP $\geq 10 \mathrm{ng} / \mathrm{mL}, \mathrm{DCP} \geq 28 \mathrm{mAU} / \mathrm{mL}, \mathrm{AFP}-\mathrm{L} 3 \geq 10 \%$ and non-SVR 12 [Table 3].

In the multivariate analysis, the factors associated with HCC recurrence were multiple tumors at the first HCC treatment $[\mathrm{HR}=2.21 ; 95 \%$ confidence interval (CI): 1.41-3.49], a history of multiple treatments for HCC before DAA therapy $(\mathrm{HR}=1.97 ; 95 \% \mathrm{CI}: 1.28-3.02)$ and AFP-L3 $\geq 10 \%$ at the initiation of DAA therapy (HR $=4.74 ; 95 \% \mathrm{CI}: 2.10-10.7)$ [Table 4].

The relationship between the number of times the patient had received treatment for HCC and recurrence is shown in [Figure 3]. The recurrence rates increased as the number of previous treatments for HCC increased: the 
Table 2. Characteristics of hepatocellular carcinoma before direct-acting antiviral treatment

\begin{tabular}{|c|c|c|c|}
\hline \multirow{2}{*}{ Characteristics } & \multirow{2}{*}{ All patients $(n=312)$} & \multicolumn{2}{|c|}{ Final HCC Tx before DAA } \\
\hline & & Hepatectomy $(n=89)$ & RFA $(n=223)$ \\
\hline \multicolumn{4}{|l|}{ At the first HCC Tx } \\
\hline Max tumor diameter $(\mathrm{mm})^{\star}$ & $18(14-25)$ & $22(16-30)$ & $16(13-22)$ \\
\hline \multirow{2}{*}{ Tumor number $(1 / 2 / \geq 3)^{\star \star}$} & $244(78.2 \%) / 42$ & $73(82.0 \%) / 14(15.7 \%) / 2$ & $171(76.7 \%) / 28$ \\
\hline & $(13.5 \%) / 26(8.3 \%)$ & $(2.3 \%)$ & $(12.6 \%) / 24(10.7 \%)$ \\
\hline $\operatorname{AFP}(\mathrm{ng} / \mathrm{mL})$ & $14.8(7.2-57.4)$ & $19.4(8.1-99.3)$ & $13.7(6.8-52.1)$ \\
\hline $\mathrm{DCP}(\mathrm{mAU} / \mathrm{mL})$ & $24.5(17-56.8)$ & $25(16-142)$ & $24(17-48)$ \\
\hline AFP-L3 (\%) & $4.3(0-7.3)$ & $6.4(0-15.8)$ & $4.3(0-6.6)$ \\
\hline \multicolumn{4}{|l|}{ At the final HCC Tx before DAA } \\
\hline Max tumor diameter $(\mathrm{mm})^{\star}$ & $15(12-21.3)$ & $22(15.5-30)$ & $15(11-20)$ \\
\hline Tumor number $(1 / 2 / \geq 3)^{\star \star}$ & $\begin{array}{l}246(78.8 \%) / 43 \\
(13.8 \%) / 23(7.4 \%)\end{array}$ & $74(83.1 \%) / 13(14.6 \%) / 2(2.3 \%)$ & $\begin{array}{l}172(77.1 \%) / 30 \\
(13.5 \%) / 21(9.4 \%)\end{array}$ \\
\hline \multicolumn{4}{|l|}{ At the initiation of DAA } \\
\hline \multirow[t]{2}{*}{ History of HCC $\operatorname{Tx}(1 / 2 / \geq 3)^{\star}$} & $190(60.9 \%) / 62$ & $82(92.1 \%) / 6(6.7 \%) / 1(1.1 \%)$ & $108(48.4 \%) / 56$ \\
\hline & $(19.9 \%) / 60(19.2 \%)$ & & $(25.1 \%) / 59(26.5 \%)$ \\
\hline The period from the final HCC Tx (days)* & $297(117-96)$ & $495(209-1101)$ & $233(104-544)$ \\
\hline $\operatorname{AFP}(\mathrm{ng} / \mathrm{mL})^{\star}$ & $8.4(4.9-16.9)$ & $7.5(4.1-11.4)$ & $9.1(5.1-23.6)$ \\
\hline $\mathrm{DCP}(\mathrm{mAU} / \mathrm{mL})^{\star \star}$ & $16.5(12-23)$ & $15(11-19)$ & $18(12-25)$ \\
\hline AFP-L3 $(\%)^{\star \star}$ & $3.5(0-5.6)$ & $0(0-4.6)$ & $3.8(0-5.8)$ \\
\hline \multicolumn{4}{|l|}{ After DAA Therapy } \\
\hline 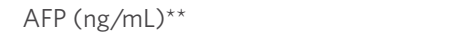 & $5.3(3.3-7.7)$ & $4.8(3.1-6.7)$ & $5.5(3.4-8.5)$ \\
\hline $\mathrm{DCP}(\mathrm{mAU} / \mathrm{mL})^{\star}$ & $17(13-23)$ & $15(12-20)$ & $18(14-24.8)$ \\
\hline AFP-L3 $(\%)^{\star \star}$ & $0(0-2)$ & $0(0-0)$ & $0(0-3.2)$ \\
\hline
\end{tabular}

${ }^{\star} P<0.001 ;{ }^{*} P<0.05 ;$ The values indicate the median (interquartile range) unless otherwise noted; AFP: alpha-fetoprotein; DCP: des-gamma-carboxy prothrombin; AFP-L3: lens culinaris agglutinin-reactive alpha-fetoprotein; HCC: hepatocellular carcinoma; Tx: treatment; DAA: direct-acting antiviral

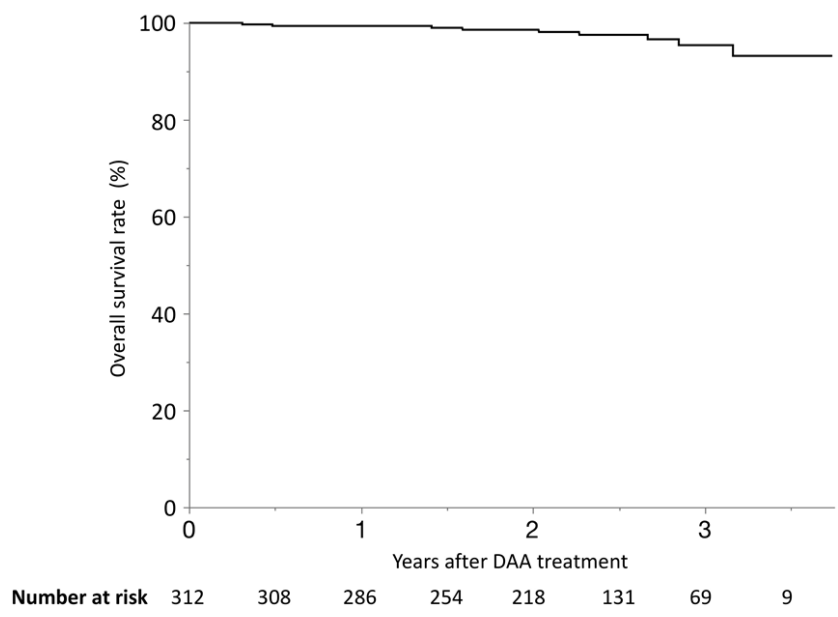

Figure 2. The overall survival in patients treated with DAAs after curative treatment for HCC. The survival rates at 1,2 and 3 years were 99\%, 98\% and 95\%, respectively. HCC: hepatocellular carcinoma; DAA: direct-acting antiviral

1-, 2- and 3-year cumulative recurrence rates after DAA therapy were $11.4 \%, 31.6 \%$ and $49.4 \%$ in patients with 1 previous treatment; $25.1 \%, 45.3 \%$ and $57.5 \%$ in patients with $2-4$ previous treatments; and $47.6 \%, 70.9 \%$ and $100 \%$ in patients with more than 4 previous treatments, respectively.

\section{Patterns of recurrence}

The patterns of HCC recurrence after DAA therapy are shown in [Table 5]. The median diameter of the tumors was $13 \mathrm{~mm}$, and 44 patients (32.6\%) had multiple tumors, including 3 patients $(2.2 \%)$ with extrahepatic metastasis. Two patients (1.5\%) had rapidly progressing tumors: 1 had massive infiltrative-growing tumors with invasion to the 
Table 3. Risk factors associated with hepatocellular carcinoma recurrence, as determined by a univariate Cox regression analysis

\begin{tabular}{|c|c|c|c|c|}
\hline Risk factors & Category & HR & $95 \% \mathrm{Cl}$ & $P$ value \\
\hline \multicolumn{5}{|l|}{ At the initiation of DAA therapy } \\
\hline Age (years) & $\geq 75(v s .<75)$ & 0.99 & $0.70-1.38$ & 0.94 \\
\hline Gender & Male (vs. female) & 1.16 & $0.82-1.63$ & 0.41 \\
\hline Platelet $\left(\times 10^{3} / \mu \mathrm{L}\right)$ & $<100(v s . \geq 100)$ & 0.93 & $0.66-1.31$ & 0.69 \\
\hline $\mathrm{ALB}(\mathrm{g} / \mathrm{dL})$ & $<3.8(v s . \geq 3.8)$ & 1.05 & $0.75-1.48$ & 0.76 \\
\hline T.Bil (mg/dL) & $>0.8(v s . \leq 0.8)$ & 1.65 & $1.17-2.33$ & $<0.01$ \\
\hline AST (U/L) & $>52(v s . \leq 52)$ & 0.95 & $0.68-1.33$ & 0.75 \\
\hline $\operatorname{ALT}(U / L)$ & $>41(v s . \leq 41)$ & 0.87 & $0.62-1.22$ & 0.41 \\
\hline GGTP (U/L) & $>37(v s . \leq 37)$ & 0.84 & $0.60-1.17$ & 0.30 \\
\hline PT-INR & $>1.0(v s . \leq 1.0)$ & 0.99 & $0.67-1.46$ & 0.95 \\
\hline Child-Pugh grade & $\mathrm{A}(v s . \mathrm{B})$ & 0.93 & $0.60-1.43$ & 0.73 \\
\hline $\operatorname{AFP}(\mathrm{ng} / \mathrm{mL})$ & $\geq 10(v s .<10)$ & 1.14 & $0.81-1.60$ & 0.46 \\
\hline $\mathrm{DCP}(\mathrm{mAU} / \mathrm{mL})$ & $\geq 28(v s .<28)$ & 1.05 & $0.67-1.64$ & 0.83 \\
\hline AFP-L3 (\%) & $\geq 10(v s .<10)$ & 5.22 & $2.39-11.4$ & $<0.01$ \\
\hline Fib-4 index & $\geq 3.25(v s .<3.25)$ & 1.26 & $0.77-2.04$ & 0.36 \\
\hline HCV genotype & Genotype 1 (vs. others) & 1.42 & $0.17-11.7$ & 0.75 \\
\hline HCV-RNA (log IU/mL) & $>6.0(v s . \leq 6.0)$ & 1.22 & $0.87-1.71$ & 0.25 \\
\hline History of HCC Tx & Multiple (vs. single) & 1.89 & $1.35-2.65$ & $<0.01$ \\
\hline The period from the final HCC Tx (year) & $\leq 1(v s .>1)$ & 1.47 & $1.04-2.09$ & 0.03 \\
\hline \multicolumn{5}{|l|}{ At the first HCC Tx } \\
\hline Maximum tumor diameter (mm) & $>20(v s . \leq 20)$ & 1.19 & $0.84-1.69$ & 0.33 \\
\hline Tumor number & Multiple (vs. single) & 2.01 & $1.39-2.90$ & $<0.01$ \\
\hline $\operatorname{AFP}(n g / m L)$ & $\geq 10(v s .<10)$ & 1.50 & $1.04-2.16$ & 0.03 \\
\hline $\mathrm{DCP}(\mathrm{mAU} / \mathrm{mL})$ & $\geq 28(v s .<28)$ & 1.24 & $0.88-1.76$ & 0.22 \\
\hline AFP-L3 (\%) & $\geq 10(v s .<10)$ & 1.23 & $0.71-2.14$ & 0.46 \\
\hline \multicolumn{5}{|l|}{ At the final HCC Tx before DAA } \\
\hline Maximum tumor diameter (mm) & $>20(v s . \leq 20)$ & 1.05 & $0.71-1.55$ & 0.80 \\
\hline Tumor number & Multiple (vs. single) & 1.72 & $1.18-2.50$ & $<0.01$ \\
\hline The final HCC Tx before DAA & RFA (vs. hepatectomy) & 1.49 & $1.00-2.22$ & 0.049 \\
\hline \multicolumn{5}{|l|}{ After DAA Therapy } \\
\hline $\operatorname{AFP}(n g / m L)$ & $\geq 10(v s .<10)$ & 1.45 & $0.93-2.25$ & 0.10 \\
\hline $\mathrm{DCP}(\mathrm{mAU} / \mathrm{mL})$ & $\geq 28(v s .<28)$ & 1.62 & $1.05-2.49$ & 0.03 \\
\hline AFP-L3 (\%) & $\geq 10(v s .<10)$ & 4.19 & $1.94-9.04$ & $<0.01$ \\
\hline SVR (12 weeks) & No (vs. yes) & 1.68 & $0.93-3.05$ & 0.09 \\
\hline
\end{tabular}

Cl: confidence interval; ALB: albumin; T.Bil: total bilirubin; AST: aspartate aminotransferase; ALT: alanine aminotransferase; GGTP: gamma-glutamyl transpeptidase; PT-INR: prothrombin time-international normalized ratio; AFP: alpha-fetoprotein; DCP: des-gamma-carboxy prothrombin; AFP-L3: lens culinaris agglutinin-reactive alpha-fetoprotein; FIB-4 index = Age (years) $\times$ AST $(U / L) /\left[P L T\left(10^{9} / L\right) \times A L T^{1 / 2}\right.$ $(\mathrm{U} / \mathrm{L})$ ]; HCV: hepatitis C virus; HCC: hepatocellular carcinoma; Tx: treatment; DAA: direct-acting antiviral; SVR: sustained virological response

Table 4. Risk factors associated with hepatocellular carcinoma recurrence, as determined by a multivariate Cox regression analysis

\begin{tabular}{llll}
\hline Risk factors & HR & $\mathbf{9 5 \%} \mathbf{C l}$ & P value \\
\hline Multiple tumors at the first HCC Tx & 2.21 & $1.41-3.49$ & $<0.01$ \\
History of multiple Tx for HCC before DAAs & 1.97 & $1.28-3.02$ & $<0.01$ \\
AFP-L3 $\geq 10 \%$ at the initiation of DAAs & 4.74 & $2.10-10.7$ & $<0.01$ \\
\hline
\end{tabular}

$\mathrm{Cl}$ : confidence interval; AFP-L3: lens culinaris agglutinin-reactive alpha-fetoprotein; HCC: hepatocellular carcinoma; Tx: treatment; DAA: direct-acting antiviral

umbilical portion of the portal vein and lung metastasis at 1 month after DAA therapy (3 months after RFA), and the other had 2 infiltrative-growing tumors of $>40$-mm diameter at 8 months after DAA therapy (15 months after RFA); they died 56 days and 15 months after recurrence, respectively. 


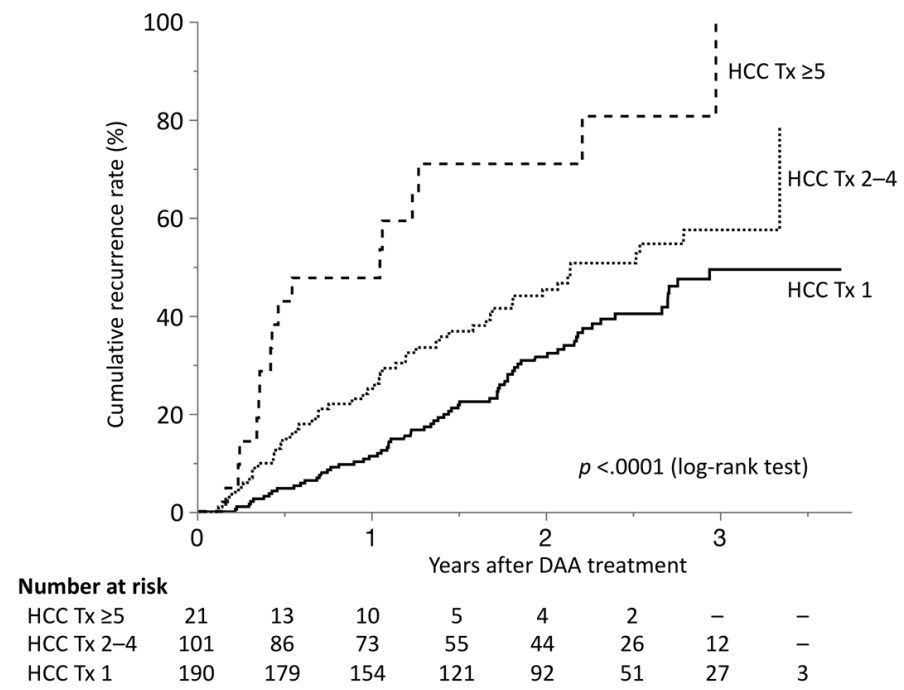

Figure 3. Recurrence in patients treated with DAAs after curative treatment for HCC according to the number of previous HCC treatments. The recurrence rates increased as the number of previous HCC treatments increased $(P<0.0001$, log-rank test). HCC: hepatocellular carcinoma; DAA: direct-acting antiviral; Tx: treatment

Table 5. Hepatocellular carcinoma status at the time of recurrence after direct-acting antiviral therapy

\begin{tabular}{lll}
\hline & \multicolumn{1}{c}{ The final HCC Tx before DAA } \\
\hline & \multicolumn{1}{c}{ Hepatectomy } & \multicolumn{1}{c}{ RFA } \\
\hline Number of patients with recurrence & 32 & 103 \\
Non-SVR & $4(12.5 \%)$ & $8(7.8 \%)$ \\
Maximum tumor diameter $(\mathrm{mm})$ & $13(10-17)$ & $13(10-17)$ \\
Tumor number $(1 / 2 / 3 / \geq 4)$ & $21(65.6 \%) / 8(25.0 \%) / 1(3.1 \%) / 2(6.3 \%)$ & $70(68.0 \%) / 19(18.4 \%) / 6(5.8 \%) / 8(7.8 \%)$ \\
Extrahepatic metastasis (positive) & $0(0 \%)$ & $3(2.9 \%)$ \\
Vascular invasion (positive) & $1(3.1 \%)$ & $1(1.0 \%)$ \\
AFP $(\mathrm{ng} / \mathrm{mL})$ & $4.9(3.1-7.1)$ & $6.4(4.3-11.2)$ \\
DCP $(\mathrm{mAU} / \mathrm{mL})$ & $20(13-27)$ & $24(18-41)$ \\
AFP-L3 $(\%)$ & $0(0-4.9)$ & $0.5(0-6.7)$ \\
\hline
\end{tabular}

The values indicate the median (interquartile range) unless otherwise noted; AFP: alpha-fetoprotein; AFP-L3: lens culinaris agglutinin-reactive alpha-fetoprotein; DCP: des-gamma-carboxy prothrombin; HCC: hepatocellular carcinoma; Tx: treatment; DAA: direct-acting antiviral

The treatment methods for HCC after DAA therapy were as follows: RFA $(n=83,61.4 \%)$, hepatectomy $(n=14$, $10.4 \%)$, TACE $(n=22,16.3 \%)$, radiation therapy $(n=3,2.2 \%)$ and particle beam, percutaneous ethanol injection, hepatic arterial infusion, systemic chemotherapy and best supportive care $(n=1$ each).

\section{DISCUSSION}

In the present study, we found that the 1- and 3-year recurrence rates of curatively treated HCC patients who received DAA therapy were $18.3 \%$ and $55.4 \%$, respectively. In contrast, the 3 -year overall survival rate was extremely high (95.4\%). In addition, we revealed that the factors associated with recurrence were multiple tumors at the first HCC treatment, a history of multiple treatments for HCC and AFP-L3 $\geq 10 \%$ at the initiation of DAA therapy.

Table 6 summarizes the published data on HCC recurrence after DAA administration in patients with an HCC treatment history. The unexpectedly high rate of HCC recurrence reported by the Spanish group raised a number of concerns and prompted a great deal of discussion ${ }^{[4]}$. According to the original paper, 3 patients died, and 16 of 55 patients $(27.6 \%)$ developed HCC recurrence after a median follow-up period of 5.7 months. However, that group 
Table 6. Summary of hepatocellular carcinoma recurrence in patients administered direct-acting antivirals after hepatocellular carcinoma treatment

\begin{tabular}{|c|c|c|c|c|}
\hline Authors & Number of patients & HCC treatment before DAA & HCC recurrence & Follow-up period \\
\hline \multirow[t]{3}{*}{ Reig et al. ${ }^{[4]}$} & 58 & Hepatectomy 20 & $16 / 58(27.6 \%)$ & 5.7 months \\
\hline & & Ablation 32 & & \\
\hline & & TACE 6 & & \\
\hline \multirow[t]{3}{*}{ Conti et al. ${ }^{[5]}$} & 59 & Hepatectomy 23 & $17 / 59(28.8 \%)$ & 24 weeks \\
\hline & & Ablation 28 & & \\
\hline & & TACE 5 & & \\
\hline \multirow[t]{2}{*}{ Nagata et al. ${ }^{[7]}$} & 83 & N/A & SVR: $22.9 \% / 3$ years & 2.3 years \\
\hline & & & Non-SVR: $40.0 \% / 3$ years & \\
\hline \multirow[t]{4}{*}{ |keda et al. ${ }^{[9]}$} & 89 & Hepatectomy 43 & $21.8 \% / 2$ years & 20.7 months \\
\hline & & RFA 38 & & \\
\hline & & TACE 4 & & \\
\hline & & PRT 4 & & \\
\hline $\operatorname{ANRS}^{[10]}$ & 189 & N/A & $8.8 \% /$ year & 20.2 months \\
\hline Minami et $a l_{.[13]}$ & 27 & RFA 27 & $29.8 \% / 2$ year & 1.3 years \\
\hline
\end{tabular}

HCC: hepatocellular carcinoma; DAA: direct-acting antiviral; TACE: transcatheter arterial chemoembolization; PRT: particle radiation therapy; RFA: radio-frequency ablation; SVR: sustained virological response

included patients treated with TACE, which was not a curative treatment; recurrence occurred only in cases treated with hepatectomy or RFA before DAA, and no recurrence was reported in cases treated with TACE, possibly because of the small number of TACE cases (only 6). Another Italian group also reported that HCC recurrence was observed in 17 of $59(28.8 \%)$ cirrhotic patients with a history of previous liver cancer during a 24-week follow-up $\operatorname{period}^{[5]}$.

In contrast to these studies, a French prospective cohort study did not observe an increased risk of HCC recurrence after DAA therapy in patients who underwent curative HCC treatment: the rates of recurrence were similar when comparing 189 patients who received DAAs (recurrence, $n=24$; incidence, 8.8\%/year) to 78 patients who did not receive DAAs (recurrence, $n=16$; incidence, $7.9 \% /$ year) ${ }^{[10]}$. A Canadian group examined the effect of HCV eradication pre- and post-liver transplantation (LT) and reported that the treatment of HCV with DAAs prior to LT $(n=13)$ enabled an SVR to be achieved in $92.3 \%$ of patients with no influence on the HCC progression or mean waiting time ${ }^{[14]}$. In 2017 , Zanetto et al. ${ }^{[15]}$ examined the dropout rate from an LT waiting list because of HCC progression in HCV-infected patients treated with DAAs. They reported that 2 of 23 DAA-treated patients (8.7\%) and 1 of 23 controls $(4.3 \%)$ were registered as dropout events due to HCC progression $(P=0.90)$ and concluded that $\mathrm{HCV}$ eradication did not seem to be associated with an increased risk of dropout from the waiting list.

Several such studies have been reported from Japan ${ }^{[7,9,13]}$. Minami et al. ${ }^{[13]}$ examined the recurrence of HCC after RFA treatment and noted no difference in the rate or aggressiveness of recurrence between cases treated withs DAA $(n=27)$ and those treated with interferon $(n=38)$ within 2 years after ablation. Ikeda et al. showed that the 1- and 2-year recurrence rates after curative treatment for HCC were $18.1 \%$ and $25.0 \%$, respectively, in patients with DAA therapy and $21.8 \%$ and $46.5 \%$, respectively, in those without DAA therapy ${ }^{[\rho]}$. Nagata et al ${ }^{[7]}$ showed that the rate of cumulative HCC recurrence in patients with an SVR after interferon-free therapy was $28.9 \%$ (22/76) during a median follow-up period of 2.3 years and concluded that the risks of early HCC recurrence after viral eradication were similar between interferon-based and interferon-free therapies. However, the populations of the studies were small, and the follow-up periods were short. We therefore tried to eliminate these problems as much as possible by increasing the number of cases and prolonging the observation period.

In the period before the DAA era at our institution, the 1-, 3- and 5-year recurrence rates of HCC patients after RFA were $23.8 \%, 56.2 \%$ and $68.0 \%$, respectively ${ }^{[19]}$. A study from Korea ${ }^{[20]}$ reported that the 1-, 3- and 5-year cumulative intrahepatic distant recurrence rates were $24.4 \%, 59.5 \%$ and $73.1 \%$, respectively. These recurrence rates were quite similar to those of our study of DAA-treated patients. Considering that the subjects in these studies were limited 
to patients with HCC at the initial treatment and that $39 \%$ of the patients in our study had recurrent HCC, we concluded that DAA treatment did not induce recurrence more frequently than interferon treatment, as we found that a history of multiple treatments for HCC before DAA therapy was an important risk factor for recurrence after DAA therapy.

We previously reported that AFP $>10 \mathrm{ng} / \mathrm{mL}$ after RFA for HCC is a significant risk factor for recurrence ${ }^{[21]}$. In the present study, however, AFP elevation before DAA treatment was not extracted as a significant factor for HCC recurrence. The discrepancy may be due to the small number of high-AFP cases in the present study due to curative treatment and the suppression of carcinogenic potential by DAA therapy. The median AFP values before and after DAA treatment were $8.4 \mathrm{ng} / \mathrm{mL}$ and $5.3 \mathrm{ng} / \mathrm{mL}$, respectively, in the present study. Of note, however: the recurrence rate was higher in patients with high AFP-L3 levels at the initiation of DAA therapy than in patients with low AFP-L3 levels. High AFP-L3 levels are known to indicate that the HCC has a high malignant carcinogenic potential and is associated with a poor prognosis ${ }^{[22]}$. Although most published studies did not report the AFP-L3 levels of their patients, it is possible that case studies showing high recurrence rates after DAA included patients with high AFP-L3 levels.

We experienced two HCC patients who showed recurrence with portal vein invasion. Considering that both patients had a history of multiple treatments and the frequency of such a recurrence pattern was not high, it is difficult to conclude whether or not DAA treatment was responsible for this type of recurrence. Careful observation will be necessary in order to confirm whether or not the prevalence of such cases will increase in the future.

Our study was associated with some limitations. First, although the observation period was relatively long among studies in which DAA therapy was administered after curative treatment of HCC, the duration was still not sufficient to estimate the long-term survival. Second, the number of deaths was extremely small; thus, the effect of DAA therapy on prolonging the survival could not be evaluated. In addition, some factors affecting the recurrence of HCC, such as alcohol consumption and coexisting diabetes mellitus, have not been well studied.

In conclusion, the recurrence rate in patients treated with DAAs after curative treatment of HCC was comparable to that before the DAA era. We also found that multiple tumors at the first HCC treatment, a history of multiple treatments for HCC, and high AFP-L3 at the time of DAA treatment were risk factors for HCC recurrence. Given that the incidence of recurrence after DAA therapy was non-negligible, a long-term follow-up is necessary to ensure a long survival, especially for patients who have risk factors for recurrence.

\section{DECLARATIONS}

\section{Acknowledgments}

The authors thank Dr. Hidenori Shiraha, Dr. Fusao Ikeda, Dr. Kenji Kuwaki, Dr. Tetsuya Yasunaka, Dr. Yuki Yasunaka, Dr. Yasuto Takeuchi, Dr. Nozomu Wada, Dr. Atsushi Oyama and Dr. Akinobu Takaki for their help in collecting data and providing meaningful advice.

\section{Authors' contributions}

Study concept and design: Nakamura S, Nouso K, Okada H, Tanaka M

Acquisition of data: Nakamura S, Onishi H, Fujioka S, Araki Y, Iwadou S, Kumada T, Toyoda H, Motomura K, Kariyama K, Kobashi H, Kaneyoshi T, Ando M, Moriya A, Taniguchi H, Morimoto Y, Hagihara H

Analysis of data: Nakamura S, Nouso K

Drafting of the manuscript: Nakamura S, Nouso K

Statistical analysis: Nakamura S

Study supervision: Nouso K, Okada H, Tanaka M 


\section{Availability of data and materials}

The original raw data used to support the findings of this study have not been made available because of the risk that will come into conflict with Personal Information Protection Law in Japan.

\section{Financial support and sponsorship}

Not applicable.

\section{Conflicts of interest}

All authors declared that there are no conflicts of interest.

\section{Ethical approval and consent to participate}

Not applicable.

\section{Consent for publication}

Not applicable.

\section{Copyright}

(c) The Author(s) 2019.

\section{REFERENCES}

1. Torre LA, Bray F, Siegel RL, Ferlay J, Lortet-Tieulent J, et al. Global cancer statistics, 2012. CA Cancer J Clin 2015;65:87-108.

2. CBD 2013 Mortality and Causes of Death Collaborators. Global, regional, and national age-sex specific all-cause and cause-specific mortality for 240 causes of death, 1990-2013: a systematic analysis for the global burden of disease study 2013. Lancet 2015;385:117-71.

3. Kanwal F, Hoang T, Kramer JR, Asch SM, Goetz MB, et al. Increasing prevalence of HCC and cirrhosis in patients with chronic hepatitis C virus infection. Gastroenterology 2011;140:1182-8.

4. Reig M, Mariño Z, Perelló C, Iñarrairaegui M, Ribeiro A, et al. Unexpected high rate of early tumor recurrence in patients with HCV-related HCC undergoing interferon-free therapy. J Hepatology 2016;65:719-26.

5. Conti F, Buonfiglioli F, Scuteri A, Crespi C, Bolondi L, et al. Early occurrence and recurrence of hepatocellular carcinoma in HCV-related cirrhosis treated with direct-acting antivirals. J Hepatol 2016;65:727-33.

6. Waziry R, Hajarizadeh B, Grebely J, Amin J, Law M, et al. Hepatocellular carcinoma risk following direct-acting antiviral HCV therapy:a systematic review, meta-analyses, and meta-regression. J Hepatol 2017;67:1204-12.

7. Nagata H, Nakagawa M, Asahina Y, Sato A, Asano Y, et al. Effect of interferon-based and -free therapy on early occurrence and recurrence of hepatocellular carcinoma in chronic hepatitis C. J Hepatol 2017;67:933-9.

8. Romano A, Angeli P, Piovesan S, Noventa F, Anastassopoulos G, et al. Newly diagnosed hepatocellular carcinoma in patients with advanced hepatitis C treated with DAAs: a prospective population study. J Hepatol 2018;69:345-52.

9. Ikeda K, Kawamura Y, Kobayashi M, Kominami Y, Fujiyama S, et al. Direct-acting antivirals decreased tumor recurrence after initial treatment of hepatitis C virus-related hepatocellular carcinoma. Dig Dis Sci 2017;62:2932-42.

10. ANRS collaborative study group on hepatocellular carcinoma (ANRS CO22 HEPATHER, CO12 CirVir and CO23 CUPILT cohorts). Electronic address: stanislas.pol@aphp.fr1. Lack of evidence of an effect of direct-acting antivirals on the recurrence of hepatocellular carcinoma: data from three ANRS cohorts. J Hepatol 2016;65:734-40.

11. Huang AC, Mehta N, Dodge JL, Yao FY, Terrault NA. Direct-acting antivirals do not increase the risk of hepatocellular carcinoma recurrence after local-regional therapy or liver transplant waitlist dropout. Hepatology 2018;68:449-61.

12. Ooka Y, Miho K, Shuntaro O, Nakamura M, Ogasawara S, et al. Prediction of the very early occurrence of HCC right after DAA therapy for HCV infection. Hepatol Int 2018;12:523-30.

13. Minami T, Tateishi R, Nakagomi R, Fujiwara N, Sato M, et al. The impact of direct-acting antivirals on early tumor recurrence after radiofrequency ablation in hepatitis C-related hepatocellular carcinoma. J Hepatol 2016;65:1272-3.

14. Emamaullee JA, Bral M, Meeberg G, Montano-Loza AJ, Bain VG, et al. HCV eradication with direct-acting antivirals does not impact HCC progression on the waiting list or HCC recurrence after liver transplantation. Can J Gastroenterol Hepatol 2019;2019:2509059.

15. Zanetto A, Shalaby S, Vitale A, Mescoli C, Ferrarese A, et al. Dropout rate from the liver transplant waiting list because of hepatocellular carcinoma progression in hepatitis C virus-infected patients treated with direct-acting antivirals. Liver Transpl 2017;23:1103-12.

16. Ioannou GN, Green PK, Berry K. HCV eradication induced by direct-acting antiviral agents reduces the risk of hepatocellular carcinoma. J Hepatol 2017; doi: 10.1016/j.jhep.2017.08.030.

17. Claudon M, Dietrich CF, Choi BI, Cosgrove DO, Kudo M, et al. Guidelines and good clinical practice recommendations for Contrast Enhanced Ultrasound (CEUS) in the liver - update 2012: a WFUMB-EFSUMB initiative in cooperation with representatives of AFSUMB, AIUM, ASUM, FLAUS and ICUS. Ultrasound Med Biol 2013;39:187-210. 
18. Bruix J, Sherman M, American Association for the Study of Liver D. Management of hepatocellular carcinoma: an update. Hepatology 2011;53:1020-2.

19. Toshimori J, Nouso K, Nakamura S, Wada N, Morimoto Y, et al. Local recurrence and complications after percutaneous radiofrequency ablation of hepatocellular carcinoma: a retrospective cohort study focused on tumor location. Acta Med Okayama 2015;69:219-26.

20. Kim YS, Lim HK, Rhim H, Lee MW, Choi D, et al. Ten-year outcomes of percutaneous radiofrequency ablation as first-line therapy of early hepatocellular carcinoma: analysis of prognostic factors. J Hepatol 2013;58:89-97.

21. Dohi C, Nouso K, Miyahara K, Morimoto Y, Wada N, et al. Potential of alpha-fetoprotein as a prognostic marker after curative radiofrequency ablation of hepatocellular carcinoma. Hepatol Res 2016;46:916-23.

22. Nouso K, Kobayashi Y, Nakamura S, Kobayashi S, Takayama H, et al. Prognostic importance of fucosylated alpha-fetoprotein in hepatocellular carcinoma patients with low alpha-fetoprotein. J Gastroenterol Hepatol 2011;26:1195-200. 